\title{
Mean-Field Description of Plastic Flow in Amorphous Solids
}

\author{
Jie $\operatorname{Lin}^{1}$ and Matthieu Wyart ${ }^{2}$ \\ ${ }^{1}$ Center for Soft Matter Research, Department of Physics, New York University, \\ New York, New York 10003, USA \\ ${ }^{2}$ Institute of Theoretical Physics, Ecole Polytechnique Federale de Lausanne (EPFL), \\ CH-1015 Lausanne, Switzerland \\ (Received 20 July 2015; published 21 January 2016)
}

\begin{abstract}
Failure and flow of amorphous materials are central to various phenomena including earthquakes and landslides. There is accumulating evidence that the yielding transition between a flowing and an arrested phase is a critical phenomenon, but the associated exponents are not understood, even at a mean-field level where the validity of popular models is debated. Here, we solve a mean-field model that captures the broad distribution of the mechanical noise generated by plasticity, whose behavior is related to biased Lévy flights near an absorbing boundary. We compute the exponent $\theta$ characterizing the density of shear transformation $P(x) \sim x^{\theta}$, where $x$ is the stress increment beyond which they yield. We find that after an isotropic thermal quench, $\theta=1 / 2$. However, $\theta$ depends continuously on the applied shear stress; this dependence is not monotonic, and its value at the yield stress is not universal. The model rationalizes previously unexplained observations and captures reasonably well the value of exponents in three dimensions. Values of exponents in four dimensions are accurately predicted. These results support the fact that it is the true mean-field model that applies in large dimensions, and they raise fundamental questions about the nature of the yielding transition.
\end{abstract}

DOI: 10.1103/PhysRevX.6.011005

\section{INTRODUCTION}

Amorphous solids such as emulsions, glasses, or sands are yield stress materials that fail and flow if a sufficient shear stress is applied. In the solid phase, plasticity can be conceived as consisting of elementary rearrangements, the so-called shear transformations [1-5]. Shear transformations are localized but display long-range elastic interactions [6] and organize dynamically into elongated highly plastic regions [4,7-10]. Above some threshold stress, failure occurs and one enters a fluid phase where a stationary flow can be maintained. In various materials, rheological properties appear to be controlled by a critical point at the yield stress $\Sigma_{c}$ where the flow stops: At that point, flow curves relating shear stress and strain rate are singular [11], and the dynamics displays long-range spatial correlations $[9,12,13]$. Despite the importance of these properties in a variety of phenomena including earthquakes and landslides, a quantitative microscopic description is lacking. As is generally the case in condensed-matter systems, one expects that the density of elementary excitations strongly affects such properties. For amorphous solids, this corresponds to the density $P(x)$ of shear transformations, characterized by the additional shear stress $x$

Published by the American Physical Society under the terms of the Creative Commons Attribution 3.0 License. Further distribution of this work must maintain attribution to the author(s) and the published article's title, journal citation, and DOI.
Subject Areas: Soft Matter, Statistical Physics

required to trigger them $[14,15]$. One empirically finds a pseudogap, i.e. $P(x) \sim x^{\theta}$, for small $x$ with $\theta>0$ [15-17]. The value of $\theta$ was argued to control the singular rheological properties and diverging length scale of flow just above the yield stress $\Sigma_{c}$ [18]. The fact that $\theta>0$ was also shown to imply crackling (system spanning avalanche-type response) in the entire solid phase $\Sigma<\Sigma_{c}$ [19], where $\Sigma$ is the applied shear stress, and the value of $\theta$ affects avalanche statistics in that regime.

Pseudogaps are commonly found in glassy systems with sufficiently long-range interactions [20]. The associated exponent is constrained by stability, as occurs in electron glasses [21], fully connected spin glasses [22-26], and hard sphere packings [27-31]. In the last two cases, the associated stability bound can be proven to be saturated (a scenario referred to as marginal stability) [20]. For amorphous solids, given the elastic coupling between shear transformations, stability toward extensive avalanches can be shown to imply $\theta>0$ [16]. However, most recent data indicate that (i) for quasistatic flows (at the yield stress), $\theta \in[0.5,0.57]$ and $\theta \in[0.35,0.43]$ in two and three dimensions, respectively $[17,18]$, (ii) right after a fast quench at zero stress, $\theta \in[0.4,0.6][15,16]$, and (iii) as the stress increases within the solid phase, $\theta$ rapidly drops initially and then slowly rises again as $\Sigma_{c}$ is approached from below [19]. Thus, the marginal stability scenario does not yield the pseudogap exponent value for amorphous solids and, moreover, cannot explain its nonmonotonic dependence on the applied stress. 
An alternative route seeks progress by considering meanfield (MF) models that would allow one to compute $\theta$ in large spatial dimension $d$, where spatial correlations between local plastic events are presumably weak. Hebraud and Lequeux (HL) [32] introduced a popular model where all shear transformations interact with each other, with a similar magnitude. A pseudogap is predicted, but one finds $\theta=1$ which is far from the values observed in two and three dimensions and does not depend on the applied stress. Lemaitre and Caroli (LC) pointed out that since elastic interactions decay as an inverse power of distance, the magnitude between two randomly chosen shear transformations is broadly distributed [14]. Including this effect led to a mean-field model that was numerically shown to display a pseudogap but not solved analytically.

We introduce a class of mean-field models that interpolate continuously between these two cases and solve them using a combination of probabilistic arguments and analysis. In our models, spatial correlations are neglected. However, the distribution of stress fluctuations generated by a local event is kept the same as finite-dimensional systems. Because of the broad distribution of mechanical noises, the variables $x$ describing the stability of shear transformations undergo biased one-dimensional Lévy flights of index $\mu$ with absorbing conditions outside a compact interval and reinsertion within this interval. The HL model corresponds to $\mu \geq 2$ (Brownian motion), whereas the more physical LC model corresponds to $\mu=1$. Our findings are that (a) for $\mu>1, \theta$ is independent of system preparation and follows $\theta=\mu / 2$ for $\mu \in(1,2]$. (b) For $\mu<1, \theta=\mu / 2$ after an isotropic $(\Sigma=0)$ quench but $\theta=0$ if $\Sigma>0$, in particular, at the yield stress $\Sigma_{c}$. (c) For the physical case $\mu=1, \theta=1 / 2$ after a quench, but $\theta$ is not universal for $\Sigma>0$ and is shown to drop immediately as $\Sigma$ increases from zero and then increases continuously with the applied stress. These predictions are confirmed numerically. They are remarkably consistent with finite-dimensional observations that were unexplained even at a qualitative level, in particular, regarding the nonmonotonicity of the pseudogap exponent with the applied shear stress. Quantitatively, the values we predict for exponents are already reasonably accurate in three dimensions and become very precise in four dimensions. These facts support that our mean-field model is the true mean-field model that applies in high spatial dimensions. A surprising consequence of our approach is that the nonuniversal value $\theta\left(\Sigma_{c}\right)$ will never reach a well-defined value above some critical dimension. Instead, it simply tends to decrease with $d$, in agreement with observations.

Beyond plasticity, to our knowledge, our model provides the first nontrivial glassy system where the violation of marginal stability can be proven (i.e., the fact that the pseudogap exponent is strictly larger than what is required by stability). Another significant by-product of our work is the classification of the asymptotic scaling behavior of a biased Lévy flight close to an absorbing boundary.
Section II introduces mean-field models. Their thermodynamics limits are worked out in Sec. III, together with a derivation of the pseudogap exponent. A physical interpretation of these results based on survival probability of biased Levy flights is presented in Sec. V. Predictions are tested numerically in Sec. VI. History dependence of the pseudogap exponents is studied in Sec. VII, while Sec. VIII investigates the role of spatial dimension. We conclude by summarizing the consequences of our work for real materials and by raising open questions.

\section{MEAN-FIELD MODELS}

Following Refs. [12,16,33-35], we describe amorphous materials as consisting of $N$ blocks, each characterized by a local shear stress $\sigma_{i}$ and a local yield stress $\sigma_{i}^{\text {th }}$, which we choose to be unity. The shear stress applied on the material is $\Sigma=\sum_{i} \sigma_{i} / N$, and we assume that $\Sigma$ is constant in time (we relax this hypothesis below). A block becomes unstable if $\left|\sigma_{i}\right|>\sigma^{\text {th }} \equiv 1$. This condition is easily expressed by defining $x_{i} \equiv 1-\sigma_{i}$, and stability corresponds to $x_{i} \in[0,2]$. If the variable $x_{i}$ exits this interval at some time $t$, the block $i$ is declared "unstable." After some constant time interval $\tau_{r}$ (describing the time for a local plastic event to occur), $\sigma_{i}$ relaxes to zero; i.e., $x_{i} \rightarrow 1$ at time $t+\tau_{r}$ (allowing for a distribution of stress drop amplitude does not affect our results below). For this choice of dynamics, $x_{i} \notin[0,2]$ are always absorbing conditions for the variable $x_{i}$. (Another popular choice of dynamics assumes that if $x_{i} \notin[0,2]$, there is a finite evaporation rate at which sites become unstable. Such a dynamic leads to an absorbing condition outside [0,2] only in the quasistatic limit. For such models, our results below must apply in that limit, as supported by the fact that the pseudogap exponent then does not depend on the choice of dynamics [16].)

Such a relaxation event corresponds to a plastic strain in site $i$ of magnitude $\Delta \epsilon_{i}=\sigma_{i} / E$, where $E$ is the shear modulus. This relaxation causes a global plastic strain $\Delta \epsilon=\Delta \epsilon_{i} / N$. Moreover, the stress in $i$ is redistributed on other sites, leading to

$$
x_{j}(l+1)=x_{j}(l)+\mathcal{G}_{j i}\left(\vec{r}_{i}-\vec{r}_{j}\right) \sigma_{i}(l),
$$

where $\mathcal{G}_{j i}\left(\vec{r}_{i}-\vec{r}_{j}\right)$ is the interaction kernel which a priori depends on the position $\vec{r}_{j}$ of the sites, and the integer $l$ numbers plastic events in chronological order. If the stress is fixed, one must have $\sum_{j \neq i} \mathcal{G}_{i j}=-\mathcal{G}_{i i}=-1$. In the following, we set $E=\tau_{r}=1$.

For amorphous solids, $\mathcal{G}(r)$ is well approximated by the Eshelby kernel, of magnitude $\mathcal{G}(r) \sim 1 / r^{d}$ and whose sign depends on the relative directions between $\left(\vec{r}_{i}-\vec{r}_{j}\right)$ and the imposed shear $[6,36,37]$. This property implies that the distribution $\rho(\Delta x)$ of kicks $\Delta x_{j}=x_{j}(l+1)-x_{j}(l)$ at each plastic event is broadly distributed since site $i$ can be close to or far from site $j$. Using $\mathcal{G}(r) \sim 1 / r^{d}$, one readily finds 
[14] that $\rho(\Delta x) \sim|\Delta x|^{-1-\mu}$ with $\mu=1$. It is straightforward to extend this result to the general case $\mathcal{G}(r) \sim 1 / r^{\alpha}$, where we find $\mu=d / \alpha$. Extending Ref. [14], mean-field models can now be constructed where the distribution of kick amplitudes preserves that of the original problem but where all sites interact statistically in an identical way. This result is achieved by replacing the prescription Eq. (1) for the relaxation of site $i$ by the new rule:

$$
\begin{aligned}
x_{i}(l+1) & =1, \\
x_{j}(l+1)-x_{j}(l) & =-\frac{1-x_{i}(l)}{N-1}+\xi_{j} .
\end{aligned}
$$

In the last equation, the first term on the right side (referred to as the drift term below) ensures conservation of stress; i.e., $\sum_{i} x_{i}(l)$ does not depend on $l$. The random variable $\xi$ has zero mean $\left\langle\xi_{i}\right\rangle=0$, and its distribution $w(\xi)$ mimics that of the finite-dimensional model:

$$
w(\xi)=\frac{A}{N}|\xi|^{-\mu-1},
$$

with a lower cutoff $\xi_{c}=(2 A / \mu)^{1 / \mu} N^{-1 / \mu}$ fixed by normalization and an upper cutoff $\xi_{m}=(2 A / \mu)^{1 / \mu}$ (such a cutoff is present in the finite-dimensional model and corresponds to the amplitude of the kick given by an adjacent site). The dynamical rule of the mean-field models is illustrated in Fig. 1.

Such mean-field models behave qualitatively like standard elastoplastic models: There exists a yield stress $\Sigma_{c}>0$ such that for $\Sigma<\Sigma_{c}$, the dynamics eventually stops, corresponding to the solid phase. For $\Sigma>\Sigma_{c}$, the dynamics never stops in the thermodynamic limit and is characterized by a rate of plastic strain $\dot{\epsilon}=N_{u}\left\langle\sigma_{u}\right\rangle / N$, where $N_{u}$ is the instantaneous number of unstable blocks and $\left\langle\sigma_{u}\right\rangle=1-\left\langle x_{u}\right\rangle$ their mean stress value when they relax. $\left\langle\sigma_{u}\right\rangle$ has both a positive contribution from $\sigma_{u}>1$ (i.e., $\left.x_{u}<0\right)$ and a negative one from $\sigma_{u}<-1$ (i.e., $x_{u}>2$ ),

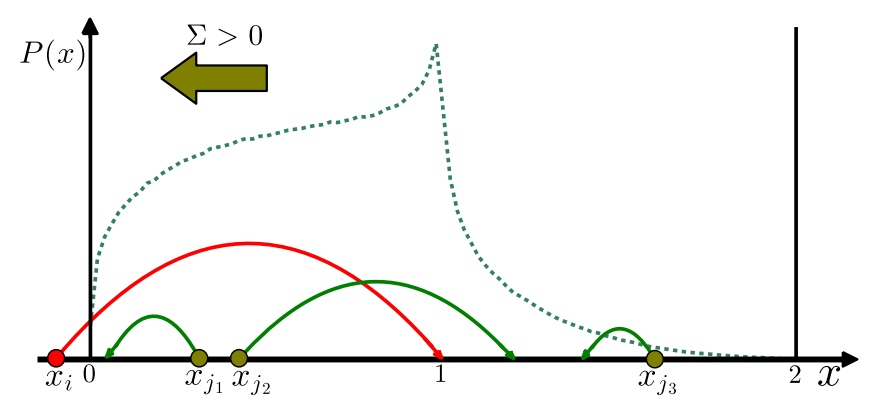

FIG. 1. Mean-field model: An unstable site (red line) returns to $x_{i}=1$ from $x_{i}<0$ after a time $\tau_{r}$. Concomitantly, other sites implement a drift toward negative $x$ to conserve the mean stress and a random jump of symmetric distribution $w(\xi)$. The dashed line represents $P(x)$, assuming $\Sigma>0$. A pseudogap is represented at $x=0$. and the symmetry between these is broken as soon as $\Sigma>0$. In our convention, for $\Sigma>0,\langle x\rangle<1$ and most sites become unstable at the boundary $x<0$, leading to $\dot{\epsilon}>0$.

Ultimately, our model describes Lévy flights with absorbing conditions for $x \notin[0,2]$. Because of the drift term in Eq. (2), these flights are biased, which tends to bring them toward the unstable region $x<0$ if $\Sigma>0$. For $\Sigma>\Sigma_{c}$, where a stationary state is reached, computing the pseudogap in this mean-field approximation requires one to obtain the stationary distribution of the stable sites $P(x) \equiv \sum_{\text {stable }} \delta\left(x-x_{i}\right) / N$ of biased Lévy flights near an absorbing boundary.

\section{CONTINUOUS DESCRIPTION}

We consider the limit $N \rightarrow \infty$ while keeping the variable $\gamma \equiv l / N$ fixed, with $\gamma \ll 1(\gamma$ is essentially a measure of the accumulated plastic strain $\epsilon$, as $\epsilon=\gamma\left\langle\sigma_{u}\right\rangle$ where, in practice, $\left\langle\sigma_{u}\right\rangle \simeq 1$ for $\Sigma \simeq \Sigma_{c}$ ). In this limit, the dynamics of stable sites in Eq. (2) becomes

$$
x_{j}(\gamma)=x_{j}(0)-v \gamma+\xi_{j}(\gamma) .
$$

Here, the drift follows:

$$
v=\left\langle\sigma_{u}\right\rangle=1-\left\langle x_{u}\right\rangle .
$$

For this convention, $v>0$ if $\Sigma>0$. We assume that $v>0$ and will relax this hypothesis when discussing thermal quenches at $\Sigma=0$. The random kick $\xi_{j}(\gamma)$ is an accumulation of $\gamma N$ discrete random kicks, $\xi_{j}(\gamma)=\sum_{k=1}^{\gamma N} \xi_{k}$, and satisfies the probability distribution

$$
w_{\gamma}(\xi)=\int \delta\left(\sum_{k=1}^{\gamma N} \xi_{k}-\xi\right) \prod_{k=1}^{\gamma N} w\left(\xi_{k}\right) d \xi_{k} .
$$

In Fourier space, $\tilde{w}_{\gamma}(k)=\tilde{w}(k)^{\gamma N}$, where $\tilde{w}(k)$ is the Fourier transform of $w(\xi)$ defined in Eq. (3).

According to Eq. (4), together with the rule that unstable sites are reinserted in $x=1$, one obtains the time evolution of the density distribution of $x, P(x)$, from $\gamma$ to $\gamma+\delta \gamma$ :

$$
\begin{aligned}
P(x, \gamma+\delta \gamma)= & P(x+v \delta \gamma, \gamma)+\int_{-\infty}^{\infty} P(y, \gamma) w_{\delta \gamma}(y-x) d y \\
& -P(x, \gamma) \int_{-\infty}^{\infty} w_{\delta \gamma}(y) d y+\delta \gamma \delta(x-1)+O\left(\delta \gamma^{2}\right),
\end{aligned}
$$

where the first term on the right side represents the drift, the second term characterizes the flux of a particle arriving in $x$, and the third term represents the flux of particles departing from $x$. Eventually, we obtain the master equation 
$\frac{\partial P}{\partial \gamma}=v \frac{\partial P(x)}{\partial x}+\int_{-\infty}^{\infty}[P(y)-P(x)] w^{\prime}(y-x) d y+\delta(x-1)$,

with the condition that $P(x)=0$ if $x \notin[0,2]$, and where $w^{\prime}(\xi)=\lim _{\gamma \rightarrow 0} w_{\gamma}(\xi) / \gamma$.

Case $\mu \geq 2$.- In this case, $w(\xi)$ has a finite variance, and $\tilde{w}(k)=1-\left\langle\xi^{2}\right\rangle k^{2} / 2+O\left(k^{4}\right)$. For $\tilde{w}_{\gamma}(k)$ to converge in the large $N$ limit, one must choose $\left\langle\xi^{2}\right\rangle=2 D / N$, where $D$ is a constant (implying $A \sim N^{1-(\mu / 2)}$ ), so $\tilde{w}_{\gamma}(k) \rightarrow$ $\exp \left(-\gamma D k^{2}\right)$. Then, $w^{\prime}(\xi)$ converges to a Gaussian, and Eq. (8) leads to the standard Fokker-Planck equation:

$$
\frac{\partial P}{\partial \gamma}=v \frac{\partial P}{\partial x}+D \frac{\partial^{2} P}{\partial x^{2}}+\delta(x-1)
$$

Solutions of such a diffusion equation vanish linearly near the absorbing condition; i.e., $P(x) \sim x$ at small $x$, as found in the HL model [32]. This corresponds to $\theta=1$.

Case $\mu<2$.- In this case, one recovers the well-known results for Lévy distribution [38]:

$$
w^{\prime}(\xi) \rightarrow \frac{A}{|\xi|^{\mu+1}}
$$

with an upper cutoff at $\xi_{m}$ (see Appendix B for details). Equation (8) then leads to

$$
\begin{aligned}
\frac{\partial P}{\partial \gamma}= & v \frac{\partial P(x)}{\partial x}-P(x) \int_{-\infty}^{0} w^{\prime}(y-x) d y \\
& +\int_{0}^{2}(P(y)-P(x)) w^{\prime}(y-x) d y+\delta(x-1),
\end{aligned}
$$

using the condition that $P(x)=0$ for $x<0$ and $x>2$.

\section{ASYMPTOTIC SOLUTIONS}

We seek stationary solutions of Eq. (11) of the form $P(x)=P_{0}+C_{0} x^{n}$ for $x \ll 1$, with $n>0$. Defining $s=y / x$, Eq. (11) reduces to

$$
\begin{aligned}
& v n C_{0} x^{n-1}-A \frac{P_{0}+C_{0} x^{n}}{\mu} x^{-\mu} \\
& +x^{n+1} \int_{0}^{2 / x}\left\{\frac{P(s x)}{x^{n}}-\frac{P_{0}}{x^{n}}-C_{0}\right\} w^{\prime}(x(s-1)) d s=0 .
\end{aligned}
$$

Here, we change the variable $s=y x$ and denote the last term $T_{3}$. Using the fact that $w^{\prime}(\xi)=A /|\xi|^{\mu+1}$, in the limit $x \ll 1, T_{3}$ converges to

$$
T_{3}=A C_{0} x^{n-\mu} \int_{0}^{\infty} \frac{s^{n}-1}{|s-1|^{\mu+1}} d s
$$

if $n<\mu$, or $T_{3}=O(1)$ if $n>\mu$.

Case $\mu<1$. - In this situation, we must have $P_{0}>0$; otherwise, the first term in Eq. (12) cannot be balanced. So $T_{3}$ is always negligible compared with the first two terms, in both cases $n<\mu$ and $n>\mu$. Keeping only the dominant terms, we obtain

$$
v n C_{0} x^{n-1}-A \frac{P_{0}}{\mu} x^{-\mu}=0,
$$

implying $n-1=-\mu$. Thus, we find

$$
P(x)=P_{0}+C_{0} x^{1-\mu},
$$

corresponding to $\theta=0$.

Case $1<\mu<2$. - In this case, if $P_{0}>0$, Eq. (12) cannot be satisfied because the term proportional to $P_{0} x^{-\mu}$ cannot be balanced. So $P_{0}=0$, and $T_{3}$ is therefore not negligible. Equating the dominant terms leads to

$$
-A \frac{C_{0}}{\mu} x^{n-\mu}+T_{3}=0 .
$$

If $n>\mu$, the first term tends to 0 , while the second term remains $O(1)$. Thus, $n \leq \mu$, and

$$
\frac{1}{\mu}-\int_{0}^{\infty} \frac{s^{n}-1}{|1-s|^{1+\mu}} d s=0 .
$$

We checked that the unique solution of this equation is $n=\mu / 2$, a result which has a simple probabilistic interpretation, as discussed in Sec. IV. Thus, $P(x) \sim x^{\mu / 2}$ and $\theta=\mu / 2$.

Case $\mu=1$. - The most important physical case is also the richest. The solution can exist only if $P_{0}=0$ and $n<1$, and Eq. (12) asymptotically implies

$$
v n x^{n-1}-A x^{n-1}+A x^{n-1} \int_{0}^{\infty} \frac{s^{n}-1}{|1-s|^{2}} d s=0 .
$$

TABLE I. Summary of results: MF values of $\theta$ at the yield stress $\Sigma_{c}$ (implying $v>0$ ) and after a quench at $\Sigma=0$ (for which $v=0)$ as a function of the Lévy index $\mu$, the random kick amplitude $A$, and the bias $v$. For comparison, we also report $\theta$ (marginality) corresponding to the saturation of the stability bound derived in Ref. [16].

\begin{tabular}{lccc}
\hline \hline$\mu$ & $\theta\left(\Sigma_{c}\right)$ & $\theta(\Sigma=0)$ & $\theta$ (Marginality) \\
\hline$\mu \geq 2$ & 1 & 1 & 1 \\
$1<\mu<2$ & $\mu / 2$ & $\mu / 2$ & $\mu-1$ \\
$\mu=1$ & $\arctan (\pi A / v) / \pi$ & $1 / 2$ & 0 \\
$\mu<1$ & 0 & $\mu / 2$ & 0 \\
\hline \hline
\end{tabular}




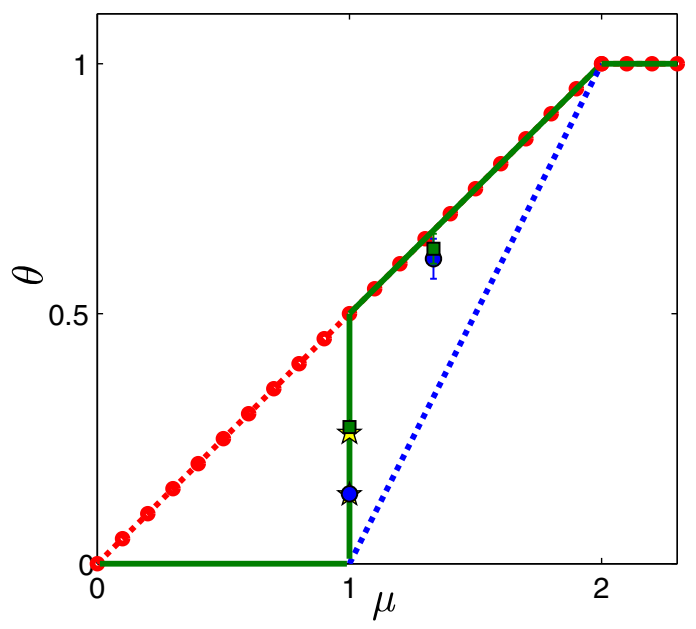

FIG. 2. Theoretical prediction of the pseudogap exponent $\theta$ vs the Lévy index of the random kicks $\mu$. Green line: $\theta$ at the yield stress $\Sigma_{c}$. Red-dotted line: $\theta$ after a quench, $\Sigma=0$, overlapping with the green line for $\mu>1$. Blue dashed line: Marginal values of $\theta$, overlapping with the green line for $\mu>2$, and $0<\mu<1$. Below the blue line, the system is unstable and forbidden dynamically [16]. The data points are the measured value of $\theta$ at $\Sigma_{c}$ in the simulated mean-field model: For $\mu=4 / 3, A=0.15$ (circle), $A=0.35$ (square); $\mu=1, A=0.15$ (circle), $A=0.35$ (square), and the yellow stars are the corresponding theoretical values indicated when $\mu=1$.

The last integral yields $I_{1}=\int_{0}^{\infty}\left(s^{n}-1 /\left|1-s^{2}\right|\right) d s=$ $1-\pi n \cot (\pi n)$, from which we obtain $v=A \pi \cot (\pi n)$. Thus, $P(x) \sim x^{\theta}$, with

$$
\theta=\frac{1}{\pi} \arctan \left(\frac{\pi A}{v}\right)
$$

implying that $\theta$ continuously depends on the drift $v$ and the magnitude of the noise $A$.

The above results are summarized in Table I and Fig. 2.

\section{INTERPRETATION}

A detailed probabilistic derivation of these results is given in Appendix A, including the case with no bias $(v=0)$. In a nutshell, $P(x)$ for $x \ll 1$ is proportional to the number of random walkers starting from $x=1$, which end up in position $x$ after a time $\gamma=O(1)$, without having crossed the absorbing condition $x<0$. For a Brownian motion (corresponding to $\mu \geq 2$ in our model), it is well known that this number vanishes linearly in $x$. This result is independent of the bias for a Brownian motion because on small length scales of order $x$ (or equivalently on small time scales of order $\gamma \sim x^{2}$ ), fluctuations always dominate the bias, which is therefore irrelevant. Fluctuations also dominate the bias for Levy flights if $\mu>1$, and thus $P(x)$ at small $x$ must again be independent of the bias, in agreement with our result that $\theta=\mu / 2$ if $\mu \in(1,2]$. The case $\mu=1$, however, is marginal: Bias and fluctuations are always comparable on all time scales, and both affect the value of
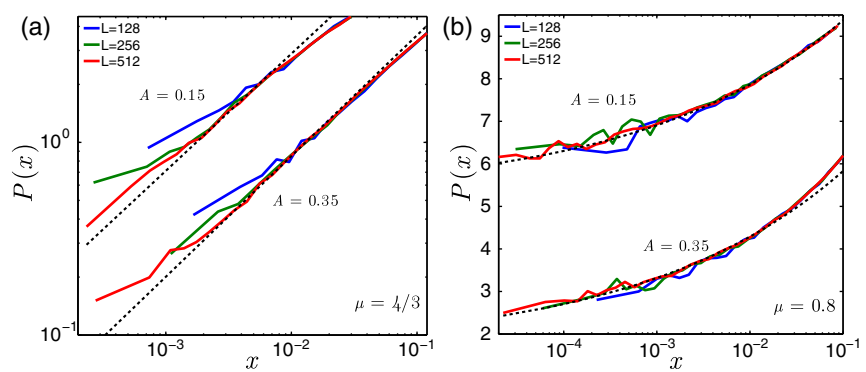

FIG. 3. $\quad P(x)$ for $\mu=4 / 3$ (a) and $\mu=0.8$ (b) for $N=L^{2}$ as shown in the legend. (a) The dashed line is the theoretical value $\theta=0.667$; the measured values are $\theta=0.61 \pm 0.04(A=0.15)$ and $\theta=0.62 \pm 0.03(A=0.35)$. (b) The dashed line is the fits of Eq. (15), which are $P(x)=5.273+6.506 x^{0.2}(A=0.15)$ and $P(x)=1.655+6.618 x^{0.2}(A=0.35)$.

$\theta$, as shown in Eq. (19). Finally, for Levy flights with $\mu<1$, the bias dominates on small time scales: Typically, walkers are essentially convected toward the origin, leading to a nonvanishing $P(x)$ at $x=0$.

\section{NUMERICAL TESTS}

We now test our predictions numerically, considering first the case $\Sigma=\Sigma_{c}>0$, implying $v>0$. To compute $P(x)$ at $\Sigma_{c}$ efficiently, we use the extremal dynamics method (see, e.g., Ref. [35]). Starting from a small value of stress, the shear stress is increased each time the dynamics stops (i.e., when there are no more unstable sites). We choose the stress increment $\delta \Sigma$ to be just sufficient to trigger a new avalanche of plasticity, i.e., $\delta \Sigma=\min \left\{x_{i}\right\}$. During avalanches, the shear stress $\Sigma$ is lowered. This is achieved, in practice, by removing the bias in Eq. (2) so that the stress drop is of approximatively $1 / N$ at each plastic event. Using such dynamics, the system spontaneously reaches the stationary state where $\langle\Sigma\rangle=\Sigma_{c}$. Fluctuations of stress vanish in the thermodynamic limit, and the trajectory of each site is equivalent to that in the fixed stress protocol at the critical stress $\Sigma_{c}$ : Both are biased Lévy flights towards the absorbing boundary with the same drift $v$. We expect our fixed stress predictions to hold, as we confirm numerically. Figure 3 shows $P(x)$ for $\mu=0.8$ and $\mu=4 / 3$ for two different choices of kick amplitude $A$. For $\mu=4 / 3$, we measure $\theta$ by fitting the part of the curves that overlap for different system sizes, and find $\theta=0.61 \pm 0.04$ $(A=0.15)$ and $\theta=0.63 \pm 0.03(A=0.35)$. These results are slightly smaller but close to the predicted value $\theta=0.667$. For $\mu=0.8$, we fit $P(x)$ by the functional form $P_{0}+C_{0} x^{1-\mu}$ predicted in Eq. (15). The fit is very good, as shown in Fig. 3(b).

For $\mu=1, \theta$ continuously depends on the kick amplitude $A$ and the bias $v$. We plot the measured value of $\theta$ and the theoretical prediction in Fig. 4, and find once again a very good agreement. 

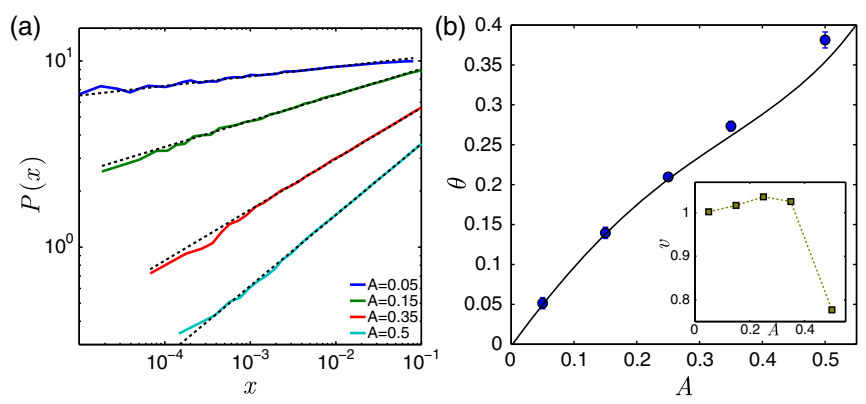

FIG. 4. (a) $P(x)$ for $\mu=1, N=512^{2}$ with different $A$. (b) Dependence of $\theta$ on $A$ for $\mu=1$. The blue dots are the numerical values extracted from (a), and the black line is the theoretical prediction Eq. (19) using the five measured values of $v$ (see inset) and a fifth-order polynominal interpolation to guide the eye.

\section{TRANSIENT BEHAVIOR}

Consider a liquid state with $\Sigma=0$. We model it as a configuration where many blocks are unstable because of thermal fluctuations. The total initial distribution (including stable and unstable sites) $P_{t}(x)$ must be symmetric around $x=1$ and display tails in the unstable regions $x<0$ and $x>2$. Next, we suddenly quenched the system by setting the temperature $T$ to zero. Importantly, the symmetry $\Sigma=0$ imposes that in the dynamics that follows, the same number of sites become unstable at $\sigma>1$ and $\sigma<-1$, implying that the drift $v=\left\langle\sigma_{u}\right\rangle=1-\left\langle x_{u}\right\rangle=0$. According to Eq. (19), we thus expect $\theta=1 / 2$ in our mean-field approximation. This prediction is consistent with the

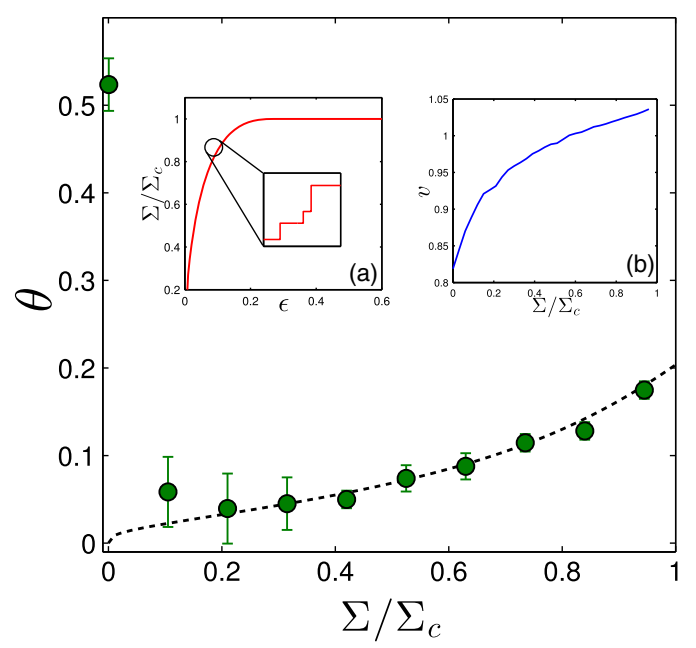

FIG. 5. $\theta$ as a function of the relative stress $\Sigma / \Sigma_{c}$, where $\Sigma_{c}$ is the yield stress for $\mu=1, A=0.3$, and $N=1024^{2}$. Right after the quench, $\theta=0.53 \pm 0.03$. As $\Sigma$ is increased, a sharp drop to a lower value occurs. Inset (a): The stress vs plastic strain curves. Zooming in on a finite-size system reveals a staircase. Inset (b): The bias $v$ 's dependence on $\Sigma / \Sigma_{c}$, from which we get the black dashed line in the main panel as the theoretical prediction using Eq. (20). molecular dynamics simulations of Ref. [15] which find $\theta \approx 0.6$ after a quench both for $d=2$ and $d=3$. It is tested numerically in our model in Fig. 5 where we find $\theta=0.53 \pm 0.03$ for an initial condition where $P_{t}(x)$ is uniform in $[-1,3]$. Numerically, we found consistent results as long as enough unstable sites are initially present.

This situation dramatically changes, however, as soon as $\Sigma$ increases from 0 . Avalanches are then triggered, and the stress vs plastic strain curves (experiments generally report the stress vs the total strain, which is a plastic strain $\epsilon$ plus an elastic contribution $\Sigma / E$ ), although smooth in the thermodynamic limit, consists of steps, as shown in inset (a) of Fig. 5 [19]. Inside these avalanches (horizontal segment in the inset), the stress is fixed and one can measure a drift $v$, as shown in inset (b) of Fig. 5. However, when the stress goes up in between avalanches [vertical segment in inset (a)], all sites are shifted toward negative $x$, leading to an additional contribution to the drift. Its magnitude in the thermodynamic limit follows $d \Sigma / d \gamma=$ $v d \Sigma / d \epsilon$. This contribution is large (and dominant) initially and vanishes at $\Sigma_{c}$ because of the shape of the stress-strain curves displayed in inset (a) of Fig. 5. Using Eq. (19), we obtain the mean-field prediction for $\theta$ in a transient:

$$
\theta=\frac{1}{\pi} \arctan \left(\frac{\pi A}{v\left(1+\frac{d \Sigma}{d \epsilon}\right)}\right)
$$

This prediction is tested in Fig. 5 and works remarkably well. Most importantly, Fig. 5 is very similar to what is found in a finite-dimensional elastoplastic model [19]. This correspondence indicates that our mean-field model correctly captures nontrivial effects present in finite dimensions that were unexplained in the past.

\section{ROLE OF SPATIAL DIMENSIONS}

In finite dimensions, the interaction kernel $\mathcal{G}$ is well described by the Eshelby kernel [6,36,37]. For $d=2$, for example, it follows that $\mathcal{G}_{j i}\left(\vec{r}_{i}-\vec{r}_{j}\right) \sim \cos (4 \phi) /\left|\vec{r}_{i}-\vec{r}_{j}\right|^{2}$, where $\phi$ is the angle between the shear direction and $\vec{r}_{i}-\vec{r}_{j}$. To quantify finite-dimensional effects, we consider the mean-field model obtained by shuffling $\mathcal{G}_{j i}$ randomly at each event:

$$
x_{j}(l+1)=x_{j}(l)+\mathcal{G}_{k_{l}(j) i}\left(\vec{r}_{i}-\vec{r}_{j}\right) \sigma_{i}(l),
$$

where $k_{l}(j)$ is a random permutation of all indices $j \neq i$. We then measure $\theta$ at $\Sigma_{c}$, considering both the twodimensional and three-dimensional Eshelby kernels, and show our results in Fig. 6(a).

We find that $\theta_{2 \mathrm{D}}^{\mathrm{SF}}=0.39 \pm 0.02$ and $\theta_{3 \mathrm{D}}^{\mathrm{SF}}=0.29 \pm 0.02$. These values are in very good agreement with the prediction in Eq. (19), $\theta_{2 \mathrm{DF}}^{\mathrm{MF}}=0.37 \pm 0.05$ and $\theta_{3 \mathrm{D}}^{\mathrm{MF}}=0.29 \pm 0.04$. To compute these predictions, we first extracted the prefactor $A_{0}$ characterizing the amplitude of the power-law distribution of 

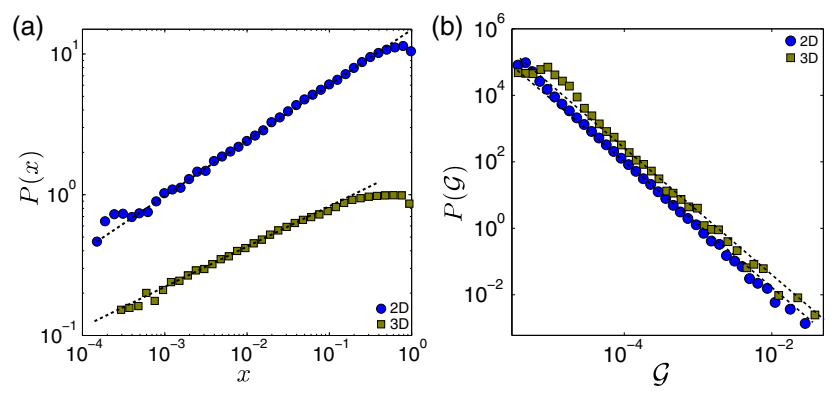

FIG. 6. (a) $P(x)$ at $\Sigma_{c}$ for the shuffled Eshelby kernel for $d=2$ and $d=3$. We find $\theta_{2 \mathrm{D}}^{\mathrm{SF}}=0.39 \pm 0.02$ and $\theta_{3 \mathrm{D}}^{\mathrm{SF}}=0.29 \pm 0.02$. (b) Direct measurements of the distribution $P(\mathcal{G})$ of the Eshelby kernel corresponding to a stress drop of unity at the origin, from which we extract $A_{0}=0.7 \pm 0.2 \quad(d=2)$ and $A_{0}=0.4 \pm 0.1(d=3)$.

the spatial kernel, as done in Fig. 6(b). Following Eq. (21), the noise amplitude $A$ is related to $A_{0}$ as $A=A_{0}\left\langle\left|\sigma_{u}\right|\right\rangle$, where $\left\langle\left|\sigma_{u}\right|\right\rangle$ is the mean absolute stress of unstable sites when they relax. The ratio $A / v$ that determines $\theta$ follows $A / v=A_{0}\left\langle\left|\sigma_{u}\right|\right\rangle /\left\langle\sigma_{u}\right\rangle$. For the mean-field model with a shuffled kernel, we numerically find that $\left\langle\left|\sigma_{u}\right|\right\rangle /\left\langle\sigma_{u}\right\rangle \approx 1$ $\left(\left\langle\left|\sigma_{u}\right|\right\rangle /\left\langle\sigma_{u}\right\rangle=1.01 \pm 5 \times 10^{-4}\right.$ in $d=2,1.0 \pm 2 \times 10^{-5}$ in $d=3$ ), a result that must hold when most sites become unstable in the direction of the shear, i.e., at the boundary $x<0$. So the result further simplifies to $A / v \approx A_{0}$.

These mean-field values for $\theta$ are systematically smaller than observations in finite dimensions where $\theta_{2 \mathrm{D}} \approx 0.57$ and $\theta_{3 \mathrm{D}} \approx 0.35[17,18]$. To complete this comparison, we computed the mean-field predictions and the finitedimensional values of $\theta$ in $d=4$ in Appendix C. Those results are summarized for different spatial dimensions in Fig. 7. We observe that the difference between the

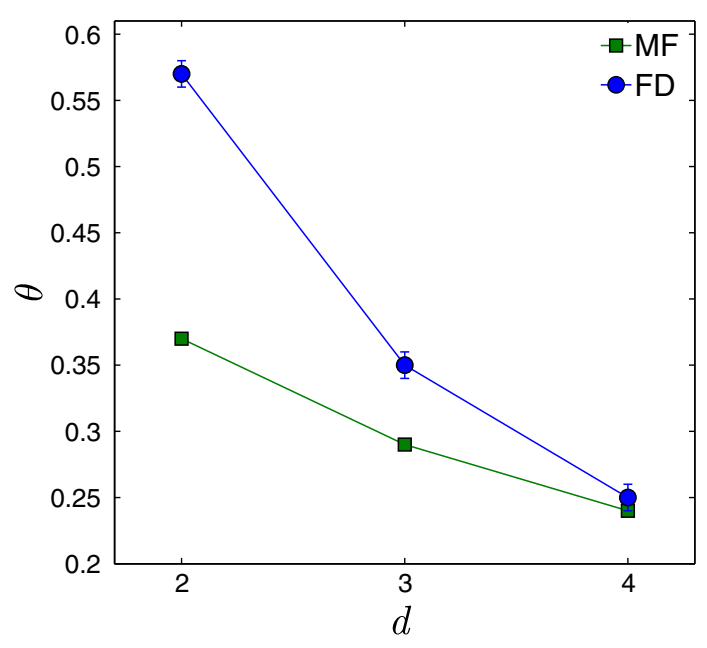

FIG. 7. Comparison between the measurements of the pseudogap exponent $\theta$ in finite-dimensional elastoplastic models (circle) and its mean-field prediction (square) as a function of the spatial dimension $d$. As $d$ increases, the difference becomes smaller and undetectable for $d=4$. mean-field prediction and finite-dimensional observations becomes smaller as the spatial dimension increases, and it becomes indistinguishable numerically for $d=4$. Our work is thus consistent with the critical dimension being $d_{c}=4$. There is currently no Ginzburg-type argument to justify why this would be the case.

\section{CONCLUSION}

We have analytically solved a mean-field model of the plasticity in amorphous solids, focusing on the exponent $\theta$ characterizing the density of shear transformations and the stability toward avalanches. The most surprising result is that $\theta$ is found to be universal after an isotropic quench, but it is otherwise stress dependent and nonuniversal. Two pieces of evidence support that our model is the correct mean-field description of elastoplasticity, for which plasticity is governed by local rearrangements interacting elastically. First, we make the surprising prediction that $\theta$ varies nonmonotonically with the stress level, a fact previously observed [19] but unexplained even at a qualitative level. Second, our prediction for $\theta$ becomes accurate as the spatial dimension increases. Most importantly, our predictions are close to observations in three dimensions. In four dimensions, predictions and observations cannot be distinguished, suggesting that the critical dimension is $d_{c}=4$.

Overall, our work is consistent with the notion that the yielding transition at $\Sigma_{c}$ is a dynamical phase transition, but it supports the fact that it is a transition of a curious kind, where exponents can depend continuously on parameters. It is still unclear if exponents in finite dimensions can be computed via a perturbation around some critical dimension, where the mean-field solution becomes exact but nonuniversal. A first step in that direction would be to build a Ginzburg-type criterion to predict the critical dimension.

It is interesting to reflect on how predictive the value of $\theta$ measured in elastoplastic models should be to describe real materials at the yield stress. From the present analysis, this is a priori not obvious at all since exponents are not expected to be universal (except at zero shear) and potentially depend on the details of the model. However, measurements in molecular dynamics simulations and elastoplastic models appear to yield very similar values for $\theta[17,18]$. A possible explanation is that in elastoplastic models, as long as most sites become unstable along the direction of the imposed shear (and not opposite to it), we predict $\theta$ to be only a function of the coefficient $A_{0}$ characterizing the Eshelby kernel $\mathcal{G}$, as discussed in Sec. VIII. The similarity between elastoplastic models and molecular dynamics simulations may thus reflect the accuracy of the Eshelby kernel in capturing the interaction between shear transformation in real amorphous solids.

Although we have focused on amorphous solids, it is very plausible that this model applies to disordered crystals as well, where plasticity is mediated by dislocations whose motions interact with the same Eshelby kernel studied here. 
It will thus be very interesting to test our predictions in both classes of materials.

Finally, the concept of marginality has been very influential in electron glasses [21] but its validity is still debated in that context [20,39]. Introducing dynamical mean-field models of the type discussed here may resolve this question.

\section{ACKNOWLEDGMENTS}

It is a pleasure to thank J. P. Bouchaud, E. DeGiuli, M. Fall, T. Gueudre, H. Hajaiej, E. Lerner, M. Muller, A. Rosso, A. Vasseur, and L. Yan for discussions related to this work, and reviewers for constructive comments. M. W. acknowledges support from NSF CBET Grant No. 1236378 and the MRSEC Program of the NSF Grant No. DMR-0820341 for partial funding.

\section{APPENDIX A: PROBABILISTIC INTERPRETATION}

We now present a probabilistic interpretation of these results. The dynamics of a single block is equivalent to a random walker with Lévy index $\mu$ in $x$ space with a bias $-v$ towards the absorbing boundary at $x=0$. We introduce the Hurst exponent for the mean absolute displacements without bias,

$$
\left\langle\left|x-x_{0}\right|\right\rangle \sim \gamma^{H},
$$

for Lévy flights $H=1 / \mu$ if $\mu<2$, and $H=1 / 2$ if $\mu \geq 2$. We define $P_{+}\left(x, x_{0},-v, \gamma\right)$ as the probability to find the walker starting from $x_{0}$ with a bias $-v$ at position $x$ after a time $\gamma$, without having crossed the $x<0$ half-line (in our argument, the presence of another wall at $x>2$ is irrelevant for the small $x$ behavior of interest here). A block that has just relaxed starts from $x_{0}=1$ and reaches $x$ after some time $\gamma$. In the stationary state, we obtain

$$
P(x)=\int_{0}^{\infty} P_{+}(x, 1,-v, \gamma) d \gamma .
$$

For small $x$, this integral is dominated by $\gamma \simeq 1$. Indeed, because $v \simeq 1$, for $\gamma \ll 1$ the walker is still far from the origin and has a negligible chance to hit the origin because of the random noise. Instead, for $\gamma \gg 1$ the walker is very likely to have entered the unstable region because of the bias. Thus, we have for small $x$,

$$
P(x) \sim P_{+}(x, 1,-v, 1) .
$$

For a Lévy flight with no bias, it is known that the absorbing boundary leads to $P_{+}\left(x, x_{0}, 0, \gamma\right) \sim x^{\theta}$ at small $x$ $[40,41]$. We define the persistence probability $S\left(x_{0}, \gamma\right)$ as the probability for the walker starting at $x_{0}>0$ to have remained in the positive axis at time $\gamma$. In the limit $x_{0} \ll \gamma^{H}$,
$S\left(x_{0}, \gamma\right) \sim \gamma^{-\kappa}$, and $\kappa$ is called the persistence exponent. There is a general scaling relation in the absence of bias, $\theta=\kappa / H$ [42]. In addition, for Lévy flights according to the Sparre Andersen theorem [43], $\kappa=\frac{1}{2}$, so for a random walker without bias $(v=0), \theta=\mu / 2$ for $\mu<2$ and $\theta=1$ for $\mu \geq 2$.

We now extend these results to the case where there is a bias. Time-reversal symmetry implies

$$
P_{+}\left(x, x_{0},-v, \gamma\right)=P_{+}\left(x_{0}, x, v, \gamma\right) \text {. }
$$

Thus, we transfer the problem to the backward process, where $x$ is now the starting position, with a drift away from the absorbing boundary. We expect $P_{+}\left(x_{0}, x, v, \gamma\right)$ to have the scaling form

$$
P_{+}\left(x_{0}, x, v, \gamma\right)=\frac{1}{\gamma^{H^{\prime}}} F\left(\frac{x_{0}}{\gamma^{H}}, \frac{x}{\gamma^{H}}, \frac{v}{\gamma^{H-1}}\right) .
$$

Integrating over $x_{0}$, we obtain the persistence probability $S(x, v, \gamma)=\gamma^{H-H^{\prime}} G\left(x / \gamma^{H}, v / \gamma^{H-1}\right)$, where $G$ is some function. Because $S(x, v, 0)=1$, we must have $H^{\prime}=H$; therefore,

$$
S(x, v, \gamma)=G\left(\frac{x}{\gamma^{H}}, \frac{v}{\gamma^{H-1}}\right) .
$$

We define the normalized surviving probability density as

$p_{x, v}\left(x_{0}, \gamma\right)=\frac{P_{+}\left(x_{0}, x, v, \gamma\right)}{\int_{0}^{\infty} P_{+}\left(x_{0}, x, v, \gamma\right) d x_{0}}=\frac{P_{+}\left(x_{0}, x, v, \gamma\right)}{S(x, v, \gamma)}$,

from which we get, together with Eq. (A5),

$$
F\left(y_{0}, y, \omega\right)=G(y, \omega) p_{y, \omega}\left(y_{0}\right)
$$

where we use the scaled variables $y=x / \gamma^{H}, \omega=v / \gamma^{H-1}$, and $p_{y, \omega}\left(y_{0}\right)=p_{x, v}\left(x_{0}, \gamma\right) \gamma^{H}$. It is clear from its definition that $p_{y, \omega}\left(y_{0}\right)$ must converge to a constant $p_{0, \omega}\left(y_{0}\right)$ as $y \rightarrow 0$. Thus, the asymptotic behavior of $F$ in the small $y$ limit is that of the surviving probability $G(y, \omega)$. From this and Eqs. (A3)-(A5), we get the central result that $P(x) \sim S(x, v, \gamma=1)$. In other words, $P(x)$ is related to the survival of a walker starting at $x$ with a positive bias $v$ after a time of order unity. According to Eq. (A6), we then have $P(x) \sim G(x, v)$, where $v$ is a constant of order one.

Case I: $1<\mu<2$. - In this case, $1 / 2<H<1$. From Eq. (A6), we get that for $y=x / \gamma^{H}$ constant and $\gamma \ll 1$, $S(x, v, \gamma)=G(y, 0)$; i.e., the drift term $v$ is irrelevant at small times. In the absence of bias, it is known that $G(y, 0) \sim y^{\mu / 2}$ [40]. As $\gamma$ increases toward 1, the effect of the bias becomes of the order of the noise; we thus expect it to only affect the survival probability by a numerical prefactor, implying that the result 


$$
\theta=\frac{\mu}{2}
$$

holds even with a finite bias, as proven above.

Case II: $\mu<1$.- In this case, $H>1$, and the bias is relevant at small times according to Eq. (A6). In that limit, $\gamma \ll 1$; we thus get $S(x, v, \gamma)=G(y, \infty)=1$. Once again, when $\gamma$ increases and becomes of order one, the effect of the noise becomes of order of the bias and will affect the survival probability by some numerical prefactor. We thus get $G(y, 1) \sim 1$ for small $y$, leading to $\theta=0$ as derived above.

Case III: $\mu=1$. - In this case, $H=1$, and the velocity is marginal in Eq. (A6), implying that $S(x, v, \gamma)=G\left(x / \gamma^{H}, v\right)$. As first derived in Ref. [44], and reviewed in Ref. [45], it is known that at long times, for $\mu=1$, the persistence exponent follows:

$$
\kappa=\frac{1}{2}-\frac{1}{\pi} \arctan (C),
$$

where $C=v / l_{\mu}$, where in our notation $l_{1}=\pi A$, leading to $C=v / \pi A$. Thus, $G\left(x / \gamma^{H}, v\right) \sim \gamma^{-\kappa}$ in that limit, which is only possible if $G\left(x / \gamma^{H}, v\right) \sim x^{\kappa / H} \sim x^{\kappa}$. This corresponds to $\theta=\kappa$. After some manipulations, it leads to

$$
\theta=\frac{1}{\pi} \arctan (\pi A / v)
$$

as derived above.

\section{APPENDIX B: ASYMPTOTIC BEHAVIOR OF $\boldsymbol{w}_{\gamma}(\boldsymbol{\xi})$}

The probability distribution of $\xi$ is

$$
w(\xi)=\frac{A}{N}|\xi|^{-\mu-1},
$$

with the lower cutoff at $|\xi|_{c}=(2 A / \mu)^{1 / \mu} N^{-(1 / \mu)}$ and the upper cutoff at $|\xi|_{m}=(2 A / \mu)^{1 / \mu}$. The corresponding Fourier transformation is

$$
\begin{aligned}
1-w(k) & =\frac{2 A}{N} \int_{\xi_{c}}^{\xi_{m}} \frac{1}{\xi^{\mu+1}}(1-\cos (k \xi)) d \xi \\
& =\frac{2 A}{N}|k|^{\mu} \int_{k \xi_{c}}^{k \xi_{m}} \frac{1}{y^{\mu+1}}(1-\cos (y)) d y
\end{aligned}
$$

in the limit $N \rightarrow \infty$; the above integral becomes

$$
\begin{aligned}
1-w(k) & =\frac{2 A}{N}|k|^{\mu} \int_{0}^{k \xi_{m}} \frac{1}{y^{\mu+1}}(1-\cos (y)) d y \\
& =\frac{2 A I_{\mu}}{N}|k|^{\mu} H\left(k \xi_{m}\right),
\end{aligned}
$$

where $I_{\mu}=-\cos (\mu \pi / 2) \Gamma(-\mu)$, and

$$
H(y)=\frac{1}{I_{\mu}} \int_{0}^{y} \frac{1-\cos (t)}{t^{\mu+1}} d t
$$

which behave as

$$
\begin{aligned}
& H(y)=1, \quad y \gg 1, \\
& H(y)=B_{0} y^{2-\mu}, \quad y \ll 1,
\end{aligned}
$$

where $B_{0}=1 /\left[2(2-\mu) I_{\mu}\right]$. In the large $N$ limit, the coarsegrained distribution $w_{\gamma}(k)$ becomes

$$
\begin{aligned}
w_{\gamma}(k) & =\left(1-\frac{2 A I_{\mu}}{N}|k|^{\mu} H\left(k \xi_{m}\right)\right)^{\gamma N} \\
& \rightarrow \exp \left\{-\gamma\left(l_{\mu}|k|\right)^{\mu} H\left(k \xi_{m}\right)\right\},
\end{aligned}
$$

where $l_{\mu}=\left(2 A I_{\mu}\right)^{1 / \mu}$. We are interested in $w_{\gamma}(\xi)$ in the limit $\gamma \rightarrow 0$, so it is convenient to define

$$
\begin{gathered}
\tilde{\xi}=\frac{\xi}{l_{\mu} \gamma^{1 / \mu}}, \\
\tilde{\xi}_{m}=\frac{\xi_{m}}{l_{\mu} \gamma^{1 / \mu}} .
\end{gathered}
$$

Making use of Eq. (B5), we can decompose $w_{\gamma}(\xi)$ as

$$
\begin{aligned}
w_{\gamma}(\xi) \approx & \frac{1}{\pi l_{\mu} \gamma^{1 / \mu}} \int_{0}^{\infty} \exp \left(-y^{\mu}\right) \cos (y \tilde{\xi}) d y \\
& +\frac{1}{\pi l_{\mu} \gamma^{1 / \mu}} \int_{0}^{\tilde{\xi}_{m}^{-1}}\left\{e^{-y^{\mu} H\left(y \tilde{\xi}_{m}\right)}-e^{-y^{\mu}}\right\} \cos (y \tilde{\xi}) d y \\
= & w_{1}+w_{2} .
\end{aligned}
$$

Here, $w_{1}=\left[1 /\left(l_{\mu} \gamma^{1 / \mu}\right)\right] \mathcal{L}_{\mu}(\tilde{\xi})$, and in the limit we are interested in, $\gamma \rightarrow 0$, it reduces to

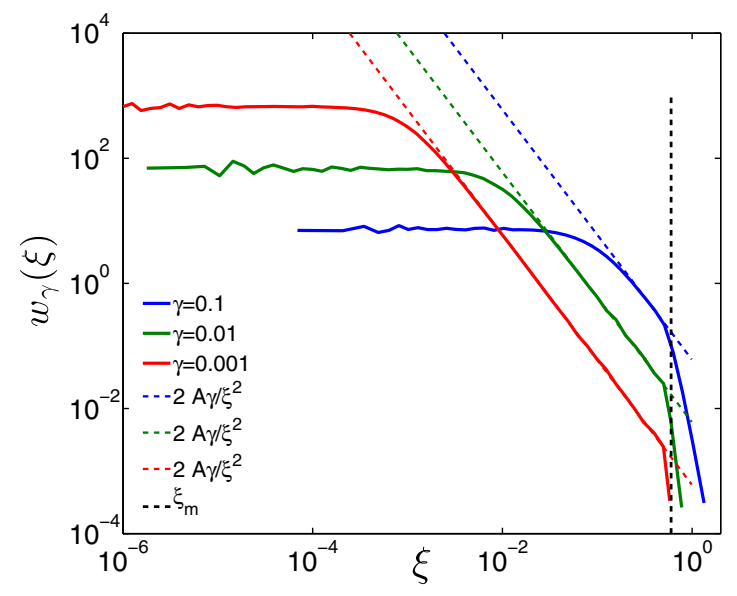

FIG. 8. Numerical calculation of $w_{\gamma}(\xi)$ at $\gamma=0.1,0.01,0.001$, $\mu=1$. The colorful dashed lines are the theoretical prediction, for $\gamma^{1 / \mu} \ll \xi \ll \xi_{m}$. The black dashed line is at $\xi_{m}$, where the upper cutoff begins to play a role. 

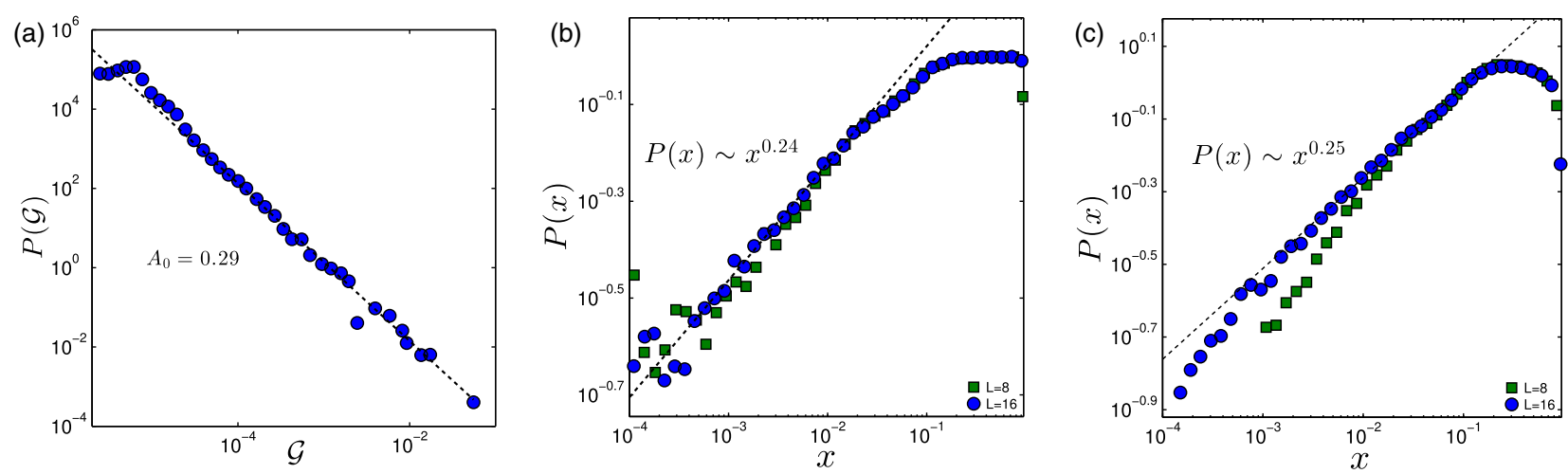

FIG. 9. (a) Direct measurements of $A_{0}$ as extracted from the distribution $P(\mathcal{G})$ of the Eshelby kernel in $d=4$. We extract $A_{0}=$ $0.29 \pm 0.4$ and predict $\theta_{4 \mathrm{DF}}^{\mathrm{MF}}=0.24 \pm 0.04$. (b) $P(x)$ at $\Sigma_{c}$ using extremal dynamics for the shuffled Eshelby kernel for $d=4$, where $\theta_{4 \mathrm{D}}^{\mathrm{SF}}=0.24 \pm 0.01$. (c) Finite-dimensions measurement of $P(x)$ in $d=4$ at fixed stress $\Sigma_{c}=0.5025$, where $\theta_{4 \mathrm{D}}=0.25 \pm 0.01$.

$$
w_{1}=\frac{\gamma A}{\xi^{\mu+1}} .
$$

We discuss the $w_{2}$ contribution in the two regimes:

Case I: $1 \ll \tilde{\xi} \ll \tilde{\xi}_{m}$. - In this regime, we can expand the exponential terms to the first order and obtain

$$
\begin{aligned}
w_{2} & \approx \frac{1}{\pi l_{\mu} \gamma^{1 / \mu}} \int_{0}^{\tilde{\xi}_{m}^{-1}}\left(y^{\mu}-B_{0} \tilde{\xi}_{m}^{2-\mu} y^{2}\right) d y \\
& =\frac{1}{\pi l_{\mu} \gamma^{1 / \mu}} \frac{2-\mu}{3(\mu+1)} \tilde{\xi}_{m}^{-\mu-1} \\
& \sim \frac{\gamma}{\xi_{m}^{\mu+1}},
\end{aligned}
$$

which turns out to be much smaller than Eq. (B9) and therefore negligible in this regime.

Case II: $1 \ll \tilde{\xi}_{m} \ll \tilde{\xi}_{\text {. }}$-In this case, we can extend the integral to infinity because of the oscillating factor,

$$
\begin{aligned}
w_{2} \approx & \frac{1}{\pi l_{\mu} \gamma^{1 / \mu}} \int_{-\infty}^{\infty}\left\{\exp \left(-B_{0} \tilde{\xi}_{m}^{2-\mu} y^{2}+i y \tilde{\xi}\right)\right. \\
& \left.-\exp \left(-|y|^{\mu}+i y \tilde{\xi}\right)\right\} d y,
\end{aligned}
$$

where the second term cancels $w_{1}$, and the resulting distribution $w_{\gamma}(\xi)$ in this limit is

$$
\begin{aligned}
w_{\gamma}(\xi) & \approx \frac{1}{l_{\mu} \gamma^{1 / \mu}} \frac{1}{\sqrt{\pi B_{0} \tilde{\xi}_{m}^{2-\mu}}} \exp \left(-\frac{\tilde{\xi}^{2}}{4 B_{0} \tilde{\xi}_{m}^{2-\mu}}\right) \\
& \sim \gamma^{-1 / 2} \exp \left(-\frac{\xi^{2}}{4 \gamma B_{0} l_{\mu}^{\mu} \xi_{m}^{2-\mu}}\right),
\end{aligned}
$$

which is much smaller than Eq. (B9) at $\xi \approx \xi_{m}$ and behaves as an upper cutoff in $w_{\gamma}(\xi)$. Our numerical calculation of $w_{\gamma}(\xi)$ is consistent with the above theoretical analysis, shown in Fig. 8.

\section{APPENDIX C: $P(x)$ IN $d=4$}

To confirm the validity of the mean field as the dimension increases, we simulate the elastoplastic model in $d=4$. Direct measurement of the coefficient $A_{0}$ characterizing the distribution $P(\mathcal{G})$ yields $A_{0}=0.29 \pm 0.05$, and numerically, we find $\left\langle\left|\sigma_{u}\right|\right\rangle /\left\langle\sigma_{u}\right\rangle=1$ without any unstable sites hitting the other absorbing boundary, from which one predicts $\theta_{4 \mathrm{D}}^{\mathrm{MF}}=0.24 \pm 0.02$, shown Fig. 9(a). For the shuffled kernel, we measure $\theta_{4 \mathrm{D}}^{\mathrm{SF}}=0.24 \pm 0.01$ [Fig. 9(b)], consistent with the theoretical prediction. The finite-dimensional measurement yields $\theta_{4 \mathrm{D}}=0.25 \pm 0.01$, as shown in Fig. 9(c).

[1] A. S Argon, Plastic Deformation in Metallic Glasses, Acta Metall. 27, 47 (1979).

[2] M.L. Falk and J.S. Langer, Dynamics of Viscoplastic Deformation in Amorphous Solids, Phys. Rev. E 57, 7192 (1998).

[3] P. Schall, D. A Weitz, and F. Spaepen, Structural Rearrangements that Govern Flow in Colloidal Glasses, Science 318, 1895 (2007).

[4] A. Amon, V. B. Nguyen, A. Bruand, J. Crassous, and E. Clément, Hot Spots in an Athermal System, Phys. Rev. Lett. 108, 135502 (2012).

[5] A. Tanguy, F. Leonforte, and J. L. Barrat, Plastic Response of a 2D Lennard-Jones Amorphous Solid: Detailed Analysis of the Local Rearrangements at Very Slow Strain Rate, Eur. Phys. J. E 20, 355 (2006).

[6] G. Picard, A. Ajdari, F. Lequeux, and L. Bocquet, Elastic Consequences of a Single Plastic Event: A Step Towards the Microscopic Modeling of the Flow of Yield Stress Fluids, Eur. Phys. J. E 15, 371 (2004).

[7] A. Le Bouil, A. Amon, J.-C. Sangleboeuf, H. Orain, P. Bésuelle, G. Viggiani, P. Chasle, and J. Crassous, A Biaxial Apparatus for the Study of Heterogeneous and Intermittent Strains in Granular Materials, Granular Matter 16, 1 (2014). 
[8] F. Gimbert, D. Amitrano, and J. Weiss, Crossover from Quasi-static to Dense Flow Regime in Compressed Frictional Granular Media, Eur. Phys. Lett. 104, 46001 (2013).

[9] A. Lemaître and C. Caroli, Rate-Dependent Avalanche Size in Athermally Sheared Amorphous Solids, Phys. Rev. Lett. 103, 065501 (2009).

[10] C. E. Maloney and M. O. Robbins, Anisotropic Power Law Strain Correlations in Sheared Amorphous 2D Solids, Phys. Rev. Lett. 102, 225502 (2009).

[11] D. Bonn, J. Paredes, M. M. Denn, L. Berthier, T. Divoux, and S. Manneville, Yield Stress Materials in Soft Condensed Matter, arXiv:1502.05281.

[12] K. Martens, L. Bocquet, and J.-L. Barrat, Connecting Diffusion and Dynamical Heterogeneities in Actively Deformed Amorphous Systems, Phys. Rev. Lett. 106, 156001 (2011).

[13] K. Michael Salerno, C. E. Maloney, and M. O. Robbins, Avalanches in Strained Amorphous Solids: Does Inertia Destroy Critical Behavior?, Phys. Rev. Lett. 109, 105703 (2012).

[14] A. Lemaître and C. Caroli, Plastic Response of a 2D Amorphous Solid to Quasi-static Shear: II-Dynamical Noise and Avalanches in a Mean Field Model, arXiv:0705.3122.

[15] S. Karmakar, E. Lerner, and I. Procaccia, Statistical Physics of the Yielding Transition in Amorphous Solids, Phys. Rev. E 82, 055103 (2010).

[16] J. Lin, A. Saade, E. Lerner, A. Rosso, and M. Wyart, On the Density of Shear Transformations in Amorphous Solids, Europhys. Lett. 105, 26003 (2014).

[17] K. Michael Salerno and M. O. Robbins, Effect of Inertia on Sheared Disordered Solids: Critical Scaling of Avalanches in Two and Three Dimensions, Phys. Rev. E 88, 062206 (2013).

[18] J. Lin, E. Lerner, A. Rosso, and M. Wyart, Scaling Description of the Yielding Transition in Soft Amorphous Solids at Zero Temperature, Proc. Natl. Acad. Sci. U.S.A. 111, 14382 (2014).

[19] J. Lin, T. Gueudré, A. Rosso, and M. Wyart, Criticality in the Approach to Failure in Amorphous Solids, Phys. Rev. Lett. 115, 168001 (2015).

[20] M. Müller and M. Wyart, Marginal Stability in Structural, Spin, and Electron Glasses, Annu. Rev. Condens. Matter Phys. 6, 177 (2015).

[21] A. L. Efros and B. I. Shklovskii, Coulomb Gap and Low Temperature Conductivity of Disordered Systems, J. Phys. C 8, L49 (1975).

[22] D. J. Thouless, P. W. Anderson, and R. G. Palmer, Solution of Solvable Model of a Spin Glass, Philos. Mag. 35, 593 (1977).

[23] F. Pázmándi, G. Zaránd, and G. T. Zimányi, Self-Organized Criticality in the Hysteresis of the Sherrington-Kirkpatrick Model, Phys. Rev. Lett. 83, 1034 (1999).

[24] P. Le Doussal, M. Müller, and K. J. Wiese, Avalanches in Mean-Field Models and the Barkhausen Noise in SpinGlasses, Europhys. Lett. 91, 57004 (2010).

[25] P. R. Eastham, R. A. Blythe, A. J. Bray, and M. A. Moore, Mechanism for the Failure of the Edwards Hypothesis in the Sherrington-Kirkpatrick Spin Glass, Phys. Rev. B 74, 020406 (2006).
[26] L. Yan, M. Baity-Jesi, M. Müller, and M. Wyart, Dynamics and Correlations Among Soft Excitations in Marginally Stable Glasses, Phys. Rev. Lett. 114, 247208 (2015).

[27] M. Wyart, Marginal Stability Constrains Force and Pair Distributions at Random Close Packing, Phys. Rev. Lett. 109, 125502 (2012).

[28] E. Lerner, G. During, and M. Wyart, Low-Energy Nonlinear Excitations in Sphere Packings, Soft Matter 9, 8252 (2013).

[29] Y. Kallus, É. Marcotte, and S. Torquato, Jammed Lattice Sphere Packings, Phys. Rev. E 88, 062151 (2013).

[30] P. Charbonneau, J. Kurchan, G. Parisi, P. Urbani, and F. Zamponi, Fractal Free Energy Landscapes in Structural Glasses, Nat. Commun. 5, 3725 (2014).

[31] P. Charbonneau, E I. Corwin, G. Parisi, and F. Zamponi, Jamming Criticality Revealed by Removing Localized Buckling Excitations, Phys. Rev. Lett. 114, 125504 (2015).

[32] P. Hébraud and F. Lequeux, Mode-Coupling Theory for the Pasty Rheology of Soft Glassy Materials, Phys. Rev. Lett. 81, 2934 (1998).

[33] J.-C. Baret, D. Vandembroucq, and S. Roux, Extremal Model for Amorphous Media Plasticity, Phys. Rev. Lett. 89, 195506 (2002).

[34] G. Picard, A. Ajdari, F. Lequeux, and L. Bocquet, Slow Flows of Yield Stress Fluids: Complex Spatiotemporal Behavior within a Simple Elastoplastic Model, Phys. Rev. E 71, 010501 (2005).

[35] M. Talamali, V. Petäjä, D. Vandembroucq, and S. Roux, Avalanches, Precursors, and Finite-Size Fluctuations in a Mesoscopic Model of Amorphous Plasticity, Phys. Rev. E 84, 016115 (2011).

[36] A. Kabla and G. Debregeas, Local Stress Relaxation and Shear Banding in a Dry Foam under Shear, Phys. Rev. Lett. 90, 258303 (2003).

[37] K. W. Desmond and E. R. Weeks, Experimental Measurements of Stress Redistribution in Flowing Emulsions, Phys. Rev. Lett. 115, 098302 (2015).

[38] J.-P. Bouchaud and A. Georges, Anomalous Diffusion in Disordered Media: Statistical Mechanisms, Models and Physical Applications, Phys. Rep. 195, 127 (1990).

[39] M. Palassini and M. Goethe, Elementary Excitations and Avalanches in the Coulomb Glass, J. Phys. Conf. Ser. 376, 012009 (2012).

[40] G. Zumofen and J. Klafter, Absorbing Boundary in OneDimensional Anomalous Transport, Phys. Rev. E 51, 2805 (1995).

[41] A. Zoia, A. Rosso, and M. Kardar, Fractional Laplacian in Bounded Domains, Phys. Rev. E 76, 021116 (2007).

[42] A. Zoia, A. Rosso, and S. N. Majumdar, Asymptotic Behavior of Self-Affine Processes in Semi-infinite Domains, Phys. Rev. Lett. 102, 120602 (2009).

[43] E. Sparre Andersen, On the Fluctuations of Sums of Random Variables II, Math. Scand. 2, 3 (1954).

[44] P. L. Doussal and K. J. Wiese, Driven Particle in a Random Landscape: Disorder Correlator, Avalanche Distribution, and Extreme Value Statistics of Records, Phys. Rev. E 79, 051105 (2009).

[45] A. J. Bray, S. N. Majumdar, and G. Schehr, Persistence and First-Passage Properties in Nonequilibrium Systems, Adv. Phys. 62, 225 (2013). 